\title{
Synthesis of Some New Nucleosides Derived From 2- Mercapto Benzimidazole with Expected Biological Activity.
}

\author{
HAMADA . H. AMER, ${ }^{1,2^{*}}$, OMAR . M . ALII, ${ }^{1 *}$ and IBRAHIM . KH . EL-KAFAWEEN ${ }^{1}$ \\ 'Department of Chemistry, Faculty of Applied and Medical Sciences, Taif University, \\ Turbah, Taif, Saudia Arabia. \\ ${ }^{2}$ Animal Medicine and Infectious Diseases Department, Faculty of Veterinary medicine, \\ Sadat City University, Egypt. \\ ${ }^{3}$ Department of Chemistry, Faculty of Science, Menoufia University, Shebin El-Koam, Egypt. \\ ${ }^{*}$ Corresponding author E-mail: dr.hamada1435@gmail.com \\ http://dx.doi.org/10.13005/ojc/330519
}

(Received: July 13, 2017; Accepted: August 01, 2017)

\begin{abstract}
2-mercaptobenzimidazole derivatives and their acyclic nucleosides were synthesized. The synthesized compounds were tested for their antibacterial activity against Escherichia coli, Staphylococcus aureus and S. epidermidis. Most of tested compounds showed moderate to high antibacterial activity while few compounds were found to show little or no activity against the tested microorganisms.
\end{abstract}

Keywords: Oxadiazolines, Arylidene derivatives, Nucleosides, Antibacterial activity

\section{INTRODUCTION}

The chemistry of heterocyclic compounds was used to prepare 2-mercaptobenzimidazole and its derivatives for obtaining novel biologically active ingredients such as anthelmintic ${ }^{1}$, anti-HIV ${ }^{2}$, antifungal ${ }^{3}$ antibacterial ${ }^{4}$, CNS depressant ${ }^{5}$, antiinflammatory ${ }^{6}$, and analgesic ${ }^{7}$ activities. In the last years, 2-mercaptobenzimidazole derivatives play an important role in therapeutic and pharmacological fields. It possesses antimicrobial ${ }^{8}$, analgesic and anti-inflammatory activities ${ }^{9}$. A number of 2-mercaptobezoimidazole derivatives have been prepared by the general method described by Van Allan and Deacon ${ }^{10}$. The synthized 2-mercaptobenzoimidazole derivatives exhibit moderate antibacterial and antifungal activities when compared to ampicillin and ketoconazole respectively ${ }^{11}$. 2-mercaptobenzimidazole derivatives were screened for their antiinflammatory activity by carrageenan induced paw edema method ${ }^{12}$. The derivatives of 2-mercaptobenzimidazole were prepared using Mannich reaction showed moderate to high antibacterial activity ${ }^{13}$. The benzimidazole moiety is present in wide antimicrobial, antiprotozoal and analgesic 
drugs $^{14-16}$. The pharmacological and biological evaluating of it is clearly concluded that the prepared compounds are promisingly high antimicrobial, anti-inflammatory and analgesic agent ${ }^{17}$. Benzimidazole derivatives play an important role in medical field with so many Pharmacological activities such as antimicrobial, antiviral, antidiabetic and anticancer activity ${ }^{18}$. Benzimidazole derivatives are considered as a promising class of biologically active heterocyclic compounds that show a wide of biological activities like anti-viral, anti-microbial, anticancer, anti-diabetic, antiparasitic, anti-oxidant, anthelmintics, anti-HIV, antiproliferative, anti-convulsant, anti-inflammatory, antihypertensive, anti-neoplastic, and anti-trichinellosis ${ }^{19}$ .Synthesized Schiff base (AMPOHA) showed hight activity against Bacillus subtilis, Escherichia coli, Aspergillu and Aspergillus flavus ${ }^{20}$.N-phthalimido and acetomido derivatives has to undergo antimicrobial activity against Klebsiella, Escherichia coli, epidermitis, Staphylococcus, Micrococcus leteus, Bacillus cereus and Staphylococcus aureus ${ }^{21}$.

\section{RESULTS AND DISCUSSION}

2-mercaptobenzimidazole (2) was synthized by mannich reaction and allowed to react with ethylchloroacetate in acetone and dry potassium carbonate to afford ethoxycarbonylmethyl-2-mercaptobenzimidazole (3) in $87 \%$ yield. Hydrazinolysis of the ethyl ester 3 in etnanol at reflux temperature affording the corresponding hydrazide 4 in $92 \%$ yield which was allowed to react with different sugars in ethanol at reflux temperature and in the presence of acetic acid to afford sugar hydrazone derivatives $5 a-d$ in $83-88 \%$ yields. Acetylation of $5 \mathrm{a}-\mathrm{d}$ with acetic anhydride in pyridine at room temperature afforded acetylated sugar hydrazone derivatives $6 a-d$ in $75-92 \%$ yields. When<smiles>Nc1ccccc1N</smiles><smiles>CCOC(=O)C(Cl)(Cl)C(=O)OCC</smiles><smiles>CCOC(CC)CCSc1nc2ccccc2[nH]1</smiles>

Scheme.1 the acetylation was carried out in acetic anhydride under reflux at $90^{\circ} \mathrm{C}$ afforded oxadiazoline derivatives $7 \mathrm{a}-\mathrm{d}$ in $70-78 \%$ yields. Treatment of hydrazide 4 with different aldehydes to give arylidene derivatives $8 \mathrm{a}-\mathrm{d}$ in $88-94 \%$ yields. The arylidene derivatives were reacted with acetic anhydride to afford oxadiazoline derivatives 9a-d in $80-88 \%$ yields.
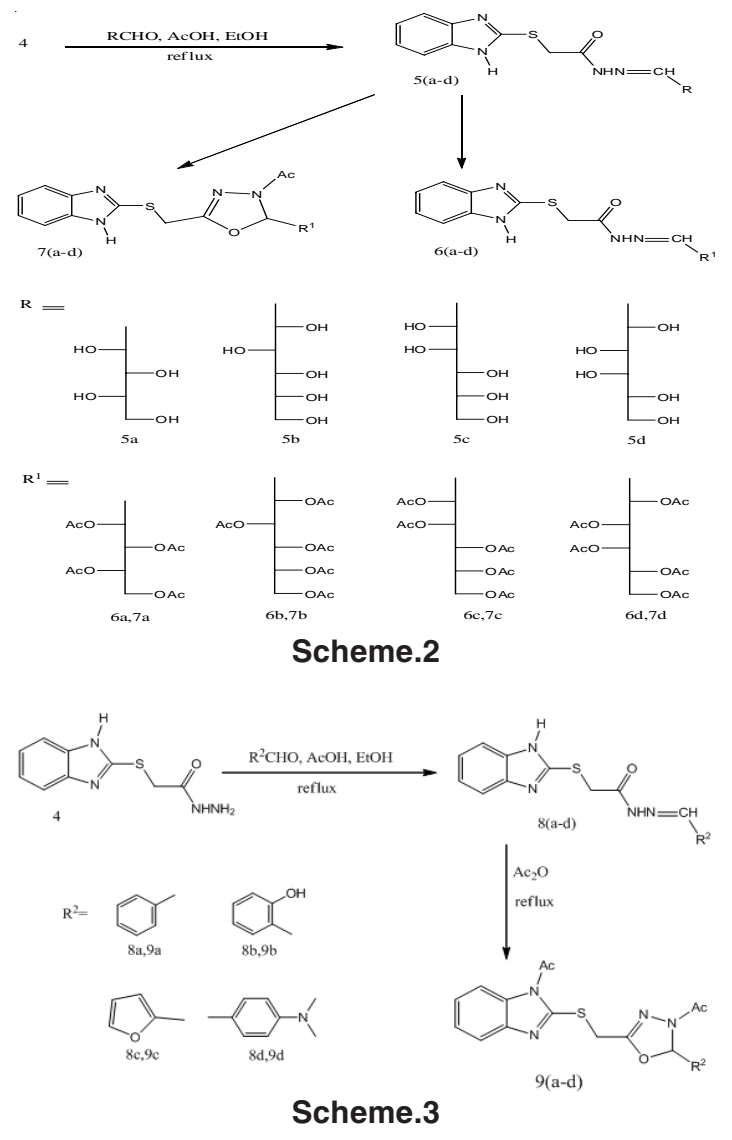

EXPERIMENTAL

Melting points were determined with a Kofler block apparatus and are uncorrected. The IR spectra were recorded on a Perkin-Elmer model 1720 FTIR spectrometer for $\mathrm{KBr}$ discs. NMR spectra were recorded on a Varian Gemini 200 NMR Spectrometer at $300 \mathrm{MHz}$ for ${ }^{1} \mathrm{H}$ NMR with TMS as a standard. The progress of the reactions was monitored by TLC using aluminum silica gel plates $60 \mathrm{~F} 245$. Spectroscopic analyses were performed at the Dr.Ahmed Farag laboratory at Faculty of science, Cairo University, Egypt. 
Ethoxycarbonylmethyl-2-mercaptobenzimidazole $(3)^{22}$.

A mixture of 2-mercaptobenzimidazole 2 (15g, $0.1 \mathrm{~mole})$, dry acetone $(300 \mathrm{ml})$, anhydrous $\mathrm{K}_{2} \mathrm{CO}_{3}$ (13.8 g, 0.1 mole) and ethylchloroacetate (15.92 g, 0.13 mole) was heated under reflux for $6 \mathrm{~h}$ (TLC). The reaction mixture was filtered of and the filtrate was evaporated under reduces pressure. The residue was recrystallized from ethanol to yield brown oil in $87 \%$ yield. $\mathrm{R}_{\mathrm{f}}=0.48(5 \% \mathrm{MeOH}$ in $\left.\mathrm{CH}_{2} \mathrm{Cl}_{2}\right) .{ }^{1} \mathrm{H}$ NMR (DMSO- $\left.\mathrm{d}_{6}\right): \mathrm{d}=1.26(\mathrm{t}, 3 \mathrm{H}, J=8.1$ $\mathrm{Hz}, \mathrm{CH}_{3} \mathrm{CH}_{2}$ ), 4.15 (q, $2 \mathrm{H}, J=8.1 \mathrm{~Hz}, \mathrm{CH}_{3} \mathrm{CH}_{2}$ ), 4.55 (s, 2H, $\mathrm{CH}_{2}$ ), 5.25 (brs, $\left.1 \mathrm{H}, \mathrm{NH}\right), 7.32$ (d, 2H, J $=5.5 \mathrm{~Hz}, \mathrm{H}-2), 7.57$ (d, $2 \mathrm{H}, \mathrm{J}=5.5 \mathrm{~Hz}, \mathrm{H}-3$ ).

\section{2-((1H-benzo[d]imidazol-2-yl)thio)acetohydrazide} $(4)^{23}$.

A mixture of 3 (2.36g, 0.01 mole), hydrazine hydrate (1.5 g, 0.03 mole) and ethanol $(40 \mathrm{ml})$ was heated under reflux for $3 \mathrm{~h}$. (TLC). The product was filtered off, recystallized from ethanol to yield white powder in 92\% yield, m.p.143-145 ${ }^{\circ} \mathrm{C} \mathrm{R}_{\mathrm{f}}=0.33\left(5 \% \mathrm{MeOH}\right.$ in $\left.\mathrm{CH}_{2} \mathrm{Cl}_{2}\right) .{ }^{1} \mathrm{H}$ NMR (DMSO$\left.\mathrm{d}_{6}\right): \mathrm{d}=2.11$ (brs, $\left.2 \mathrm{H}, \mathrm{NH}_{2}\right), 4.50\left(\mathrm{~s}, 2 \mathrm{H}, \mathrm{CH}_{2}\right), 5.35$ (brs, 1H, NH), 7.27 (d, 2H, J=5.5 Hz, Ar-H), 7.55 (d, $2 \mathrm{H}, \mathrm{J}=5.5 \mathrm{~Hz}, \mathrm{Ar}-\mathrm{H}$ ), 8.35 (brs, $1 \mathrm{H}, \mathrm{NH}$ ).

Reaction of (4) with Different sugars to Afford the Corresponding sugar hydrazones 5( a-d ).

To solution of $4(10 \mathrm{mmol})$ in absolute ethanol, different sugars $(10 \mathrm{mmol})$ were added and then glacial acetic acid $(1 \mathrm{ml})$ was added to the reaction mixture which was refluxed for $10 \mathrm{~h}$ (TLC). The solvent was evaporated or concentrated under reduced pressure and the product was filtered off to afford $5(\mathrm{a}-\mathrm{d})(83-88 \%)$ yields.

L-(-)-Arabinose(2-mercaptobenzimidazole-1-1yl) acetohydrazide (5a)

Yellow powder (83\%), m.p.182-184 ${ }^{\circ} \mathrm{C}$. $\mathrm{R}_{\mathrm{f}}$ $=0.65\left(5 \% \mathrm{MeOH}\right.$ in $\left.\mathrm{CH}_{2} \mathrm{Cl}_{2}\right),{ }^{1} \mathrm{H}$ NMR $\left(\mathrm{DMSO}-\mathrm{d}_{6}\right)$ : $\delta=3.12-3.85(\mathrm{~m}, 5 \mathrm{H}, \mathrm{H}-22, \mathrm{H}-32, \mathrm{H}-42, \mathrm{H}-52, \mathrm{H}-$ 522), 2.75 (brs, $1 \mathrm{H}, \mathrm{OH}), 3.52-3.61$ (brs, 3H, 3xOH), 4.05 (s, 2H, $\left.\mathrm{CH}_{2}\right), 4.95$ (brs, 1H, NH), 6.95-7.32 (m, $4 \mathrm{H}, \mathrm{Ar}-\mathrm{H}), 7.40$ (brs, $1 \mathrm{H}, \mathrm{NH}), 7.55$ (s, $1 \mathrm{H}, \mathrm{CH})$.

\section{D-(-)-Glucose(2-mercaptobenzimidazole-1-1-yl) acetohydrazide (5b)}

White powder (85\%), m.p.202-204 ${ }^{\circ} \mathrm{C} . \mathrm{R}_{\mathrm{f}}$ $=0.65\left(5 \% \mathrm{MeOH}\right.$ in $\left.\mathrm{CH}_{2} \mathrm{Cl}_{2}\right) \cdot{ }^{1} \mathrm{H}$ NMR $\left(\right.$ DMSO- $\left.\mathrm{d}_{6}\right): \delta$
= 3.14-3.92 (m, 5H, H-32, H-42, H-52, H-62, H-622), 3.10 (m, 1H, H-22), 3.42 (brs, 1H, OH), 3.55 (brs, $2 \mathrm{H}, 2 \mathrm{xOH}$ ), 3.62 (brs, 2H, 2xOH), 4.15 (s, 2H, CH2), 4.90 (brs, $1 \mathrm{H}, \mathrm{NH}), 6.90$ (brs, 1H, NH), 6.93-7.35 (m, 4H, Ar-H), $7.55(\mathrm{~s}, 1 \mathrm{H}, \mathrm{CH})$.

\section{D-(-)-Mannose(2-mercaptobenzimidazole-1-1-yl)} acetohydrazide $(5 \mathrm{c})$

White powder (90\%), m.p.196-198 ${ }^{\circ} \mathrm{C} . \mathrm{R}_{\mathrm{f}}$ $=0.72\left(5 \% \mathrm{MeOH}\right.$ in $\left.\mathrm{CH}_{2} \mathrm{Cl}_{2}\right) .{ }^{1} \mathrm{H}$ NMR $($ DMSO-d $): \delta$ = 3.16-3.95 (m, 5H, H-3', H-4', H-5',H-6',H-6", 3.12 (m, 1H, H-2', 3.45 (brs, $1 \mathrm{H}, \mathrm{OH}), 3.60$ (brs, $2 \mathrm{H}$, $2 \mathrm{xOH}$ ), 3.62 (brs, 2H, 2xOH), 4.15 (s, 2H, CH2), 4.90 (brs, $1 \mathrm{H}, \mathrm{NH}), 6.93$ (brs, $1 \mathrm{H}, \mathrm{NH}), 6.95-7.40$ (m, 4H, Ar-H), 7.55 (s, 1H, CH).

\section{D-(-)-Galactose(2-mercaptobenzimidazole-1-1-} yl) acetohydrazide (5d)

White powder (90\%), m.p.174-176 ${ }^{\circ} \mathrm{C} . \mathrm{R}_{\mathrm{f}}=$ $0.72\left(5 \% \mathrm{MeOH}\right.$ in $\left.\mathrm{CH}_{2} \mathrm{Cl}_{2}\right) .{ }^{1} \mathrm{H}$ NMR (DMSO-d $)$ : d = 3.13-3.94 (m, 5H, H-32, H-42, H-52, H-62, H-622), 3.13 (m, 1H, H-22), 3.44 (brs, 1H, OH), 3.59 (brs, $2 \mathrm{H}, 2 \mathrm{xOH}), 3.61$ (brs, 2H, 2xOH), 4.15 (s, 2H, CH2), 4.92 (brs, 1H, NH), 6.92 (brs, 1H, NH), 6.94-7.38 (m, 4H, Ar-H), 7.60 (s, 1H, CH).

General procedure for preparation of sugar of tetraO-acetyl- and penta-O-acetyl(2-mercaptobenzimidazol- 1-yl)acetylhydrazones 6 (a-d).

A mixture of the sugar hydrazones 5 (a-d) $(10 \mathrm{mmol})$, was dissolved in $30 \mathrm{ml}$ of pyridine and then $(15 \mathrm{mmol})$ of acetic anhydride was added. The reaction was stirred at room temperature overnight (TLC). The mixture was cooled and poured on crushed ice.The precipitate was filtered off and dried to give 6 (a- d) in $75-92 \%$ yields.

2,3,4,5-Tetra-O-acetyl-L-(-)-arabinose(2mercaptobenzimidazol-1-yl)acetylhydrazone (6a ).

Yellow gum (75\%), $\mathrm{R}_{\mathrm{f}}=0.72(5 \% \mathrm{MeOH}$ in $\mathrm{CH}_{2} \mathrm{Cl}_{2}$ ). ${ }^{1} \mathrm{H}$ NMR (DMSO-d $): \delta=1.99,2.01,2.10$, 2.21 (4s, 12H, 4xCH3CO), 4.15 (s, 2H, CH2), 4.30,4.41 (2m, 2H, H-52, H-522), $4.60(\mathrm{~m}, 1 \mathrm{H}, \mathrm{H}-$ 22), 5.06 ( brs, $1 \mathrm{H}, \mathrm{NH}$ ), $5.10(\mathrm{~m}, 1 \mathrm{H}, \mathrm{H}-42), 5.12$ (m, 1H, H-32), 7.12 ( brs, $1 \mathrm{H}, \mathrm{NH}$ ), 7.52 (d, 1H, $\mathrm{J}=2.5 \mathrm{~Hz}, \mathrm{H}-12)$, 7.25-7.62 (m,4H, Ar-H).

2,3,4,5-Tetra-O-acetyl-D-(+)-Glucose(2mercaptobenzimidazol-1-yl)acetylhydrazone ( 6b ). Yellow gum (80\%), $\mathrm{R}_{\mathrm{f}}=0.72(5 \% \mathrm{MeOH}$ in 
$\mathrm{CH}_{2} \mathrm{Cl}_{2}$ ). ${ }^{1} \mathrm{H}$ NMR (DMSO-d $)$ : $\mathrm{d}=1.97,2.03,2.04,2.06$, $2.25(5 \mathrm{~s}, 15 \mathrm{H}, 5 \times \mathrm{CH} 3 \mathrm{CO}), 4.13(\mathrm{~s}, 2 \mathrm{H}, \mathrm{CH} 2), 4.25,4.47$ (2m, 2H, H-62,H-622), 4.60 (m, 1H, H-22), 5.00 (m, 1H, $\mathrm{H}-32), 5.05$ (brs, 1H, NH), 5.10 (m, 1H, H-42), $5.12(\mathrm{~m}$, $1 \mathrm{H}, \mathrm{H}-52$ ), 7.12 ( brs, $1 \mathrm{H}, \mathrm{NH}), 7.25-7.58(\mathrm{~m}, 4 \mathrm{H}, \mathrm{Ar}-\mathrm{H})$, $7.60(\mathrm{~d}, 1 \mathrm{H}, \mathrm{J}=2.5 \mathrm{~Hz}, \mathrm{H}-12)$.

2,3,4,5-Tetra-O-acetyI-D-(+)-Mannose(2mercaptobenzimidazol-1-yl)acetylhydrazone (6c)

Yellow gum (85\%), $\mathrm{R}_{\mathrm{f}}=0.72(5 \% \mathrm{MeOH}$ in $\mathrm{CH}_{2} \mathrm{Cl}_{2}$ ). ${ }^{1} \mathrm{H}$ NMR (DMSO-d $\mathrm{d}_{6}$ ): $\delta=2.11,2.18,2.20$, 2.22, $2.26(5 \mathrm{~s}, 15 \mathrm{H}, 5 \times \mathrm{CH} 3 \mathrm{CO}), 4.11(\mathrm{~s}, 2 \mathrm{H}, \mathrm{CH} 2)$, 4.29,4.47 (2m, 2H, H-62,H-622), $4.57(\mathrm{~m}, 1 \mathrm{H}, \mathrm{H}-$ 22), $5.04(\mathrm{~m}, 1 \mathrm{H}, \mathrm{H}-32), 5.09$ (brs, $1 \mathrm{H}, \mathrm{NH}), 5.13(\mathrm{~m}$, $1 \mathrm{H}, \mathrm{H}-42), 5.20$ (m, 1H, H-52), 7.19 ( brs, $1 \mathrm{H}, \mathrm{NH}$ ), 7.29-7.59 (m, 4H, Ar-H), $7.65(\mathrm{~d}, 1 \mathrm{H}, \mathrm{J}=2.5 \mathrm{~Hz}, \mathrm{H}-$ 12).

2,3,4,5-Tetra-O-acetyl-D-(+)-Galactose(2mercaptobenzimidazol-1-yl)acetylhydrazone ( $6 \mathrm{~d}$ )

Brown gum (92\%), $\mathrm{R}_{\mathrm{f}}=0.72(5 \% \mathrm{MeOH}$ in $\mathrm{CH}_{2} \mathrm{Cl}_{2}$ ). ${ }^{1} \mathrm{H}$ NMR (DMSO-d ) $_{6}$ : $\delta=2.16,2.21,2.24$, 2.26, $2.29(5 \mathrm{~s}, 15 \mathrm{H}, 5 \mathrm{xCH} 3 \mathrm{CO}), 4.11(\mathrm{~s}, 2 \mathrm{H}, \mathrm{CH} 2)$, 4.27,4.49 (2m, 2H, H-62,H-622), $4.58(\mathrm{~m}, 1 \mathrm{H}, \mathrm{H}-$ 22), $5.03(\mathrm{~m}, 1 \mathrm{H}, \mathrm{H}-32), 5.07$ (brs, $1 \mathrm{H}, \mathrm{NH}), 5.12(\mathrm{~m}$, $1 \mathrm{H}, \mathrm{H}-42), 5.16(\mathrm{~m}, 1 \mathrm{H}, \mathrm{H}-52), 7.17$ ( brs, $1 \mathrm{H}, \mathrm{NH})$, 7.27-7.57 (m, 4H, Ar-H), $7.63(\mathrm{~d}, 1 \mathrm{H}, \mathrm{J}=2.5 \mathrm{~Hz}, \mathrm{H}-$ 12).

General procedure for the synthesis of 4-acetyl5-(tetra-and penta-0-acetylalditolyl)-2-(2mercaptobenzimidazol-1-yl)-1, 3, 4-oxadiazoline 7 ( a-d)

A solution of sugar hydrazones $5(a-d)(0$. $01 \mathrm{~mol})$ in acetic anhydride $(10 \mathrm{ml})$ was heated at $90{ }^{\circ} \mathrm{C}$ for $3 \mathrm{~h}$. The resulting solution was poured onto crushed-ice and the product that separated out was filtered off, washed with water, and then dried. The products were recrystallized from methanol to give $7(\mathrm{a}-\mathrm{d})$ in $70-78$ yields.

4 -Acetyl-5-(1,2,3,4-tetra-O-acetyl-Larabinotetritolyl)-2-(2mercaptobenzimidazol-1yl)-1,3,4-oxadiazoline (7a)

Brown oil $(70 \%), R_{f}=0.72(5 \% \mathrm{MeOH}$ in $\mathrm{CH}_{2} \mathrm{Cl}_{2}$ ). ${ }^{1} \mathrm{H}$ NMR (DMSO-d $)$ : $\delta=2.03,2.05,2.07$, 2.10, $2.21(5 \mathrm{~s}, 15 \mathrm{H}, 5 \times \mathrm{CH} 3 \mathrm{CO}), 4.40(\mathrm{~s}, 2 \mathrm{H}, \mathrm{CH} 2)$, 4.12, 4.45 (2m, 2H, H-42,H-422), 5.05 (brs, $1 \mathrm{H}, \mathrm{NH}$ ), $5.14(\mathrm{~m}, 1 \mathrm{H}, \mathrm{H}-32), 5.19(\mathrm{~m}, 1 \mathrm{H}, \mathrm{H}-22), 5.89$ (dd,
$1 \mathrm{H}, \mathrm{J}=3.2,6.2 \mathrm{~Hz}, \mathrm{H}-12), 5.89(\mathrm{~d}, 1 \mathrm{H}, \mathrm{J}=8.8 \mathrm{~Hz}$, oxadiazoline $\mathrm{H}-5)$, 7.24-7.59 (m, $4 \mathrm{H}, \mathrm{Ar}-\mathrm{H})$.

4-Acetyl-5-(1,2,3,4,5-penta-0-acetyl-Dglucopentitolyl)-2(2-methylbenzimidazol-1-yl)1,3,4-oxadiazoline ( $7 \mathrm{~b}$ )

Brown oil $(72 \%), \mathrm{R}_{\mathrm{f}}=0.72(5 \% \mathrm{MeOH}$ in $\mathrm{CH}_{2} \mathrm{Cl}_{2}$ ). ${ }^{1} \mathrm{H}$ NMR (DMSO-d 6 ): $\delta=2.04,2.07,2.09$, 2.12, 2.22, $2.34(6 \mathrm{~s}, 18 \mathrm{H}, 6 \times \mathrm{CH} 3 \mathrm{CO}), 4.60$ (s, $2 \mathrm{H}$, $\mathrm{CH} 2), 4.27,4.48$ (2m, 2H, H-52,H-522), 5.10 (brs, $1 \mathrm{H}, \mathrm{NH}),, 5.15(\mathrm{~m}, 1 \mathrm{H}, \mathrm{H}-42), 5.21(\mathrm{~m}, 1 \mathrm{H}, \mathrm{H}-32)$, $5.28(\mathrm{~m}, 1 \mathrm{H}, \mathrm{H}-22), 5.32(\mathrm{dd}, 1 \mathrm{H}, \mathrm{J}=3.2,6.2 \mathrm{~Hz}, \mathrm{H}-$ 12), $5.93(\mathrm{~d}, 1 \mathrm{H}, \mathrm{J}=8.8 \mathrm{~Hz}$, oxadiazoline $\mathrm{H}-6), 7.25$ $7.60(\mathrm{~m}, 4 \mathrm{H}, \mathrm{Ar}-\mathrm{H})$.

4-Acetyl-5-(1,2,3,4,5-penta-0-acetyl-Dglucopentitolyl)-2(2-methylbenzimidazol-1-yl)1,3,4-oxadiazoline ( 7c )

Brown oil $(73 \%), \mathrm{R}_{\mathrm{f}}=0.72(5 \% \mathrm{MeOH}$ in $\mathrm{CH}_{2} \mathrm{Cl}_{2}$ ). ${ }^{1} \mathrm{H}$ NMR (DMSO- $\left.\mathrm{d}_{6}\right): \delta=1.97,1.99,2.03$, 2.06, 2.10, $2.32(6 \mathrm{~s}, 18 \mathrm{H}, 6 \times \mathrm{CH} 3 \mathrm{CO}), 4.60(\mathrm{~s}, 2 \mathrm{H}$, $\mathrm{CH} 2), 4.24,4.46$ (2m, 2H, H-52,H-522), 5.12 (brs, $1 \mathrm{H}, \mathrm{NH}),, 5.16(\mathrm{~m}, 1 \mathrm{H}, \mathrm{H}-42), 5.22(\mathrm{~m}, 1 \mathrm{H}, \mathrm{H}-32)$, $5.26(\mathrm{~m}, 1 \mathrm{H}, \mathrm{H}-22), 5.34(\mathrm{dd}, 1 \mathrm{H}, \mathrm{J}=3.2,6.2 \mathrm{~Hz}, \mathrm{H}-$ 12), $5.94(\mathrm{~d}, 1 \mathrm{H}, \mathrm{J}=8.8 \mathrm{~Hz}$, oxadiazoline $\mathrm{H}-6), 7.22-$ $7.52(\mathrm{~m}, 4 \mathrm{H}, \mathrm{Ar}-\mathrm{H})$.

4-Acetyl-5-(1,2,3,4,5-penta-0-acetyl-Dglucopentitolyl)-2(2-methylbenzimidazol-1-yl)1,3,4-oxadiazoline (7d)

Brown oil $(78 \%), \mathrm{R}_{\mathrm{f}}=0.72(5 \% \mathrm{MeOH}$ in $\mathrm{CH}_{2} \mathrm{Cl}_{2}$ ). ${ }^{1} \mathrm{H}$ NMR (DMSO-d $\mathrm{d}_{6}$ ): $\delta=1.95,1.97,1.99$, $2.04,2.07,2.34(6 \mathrm{~s}, 18 \mathrm{H}, 6 \times \mathrm{CH} 3 \mathrm{CO}), 4.58(\mathrm{~s}, 2 \mathrm{H}$, $\mathrm{CH} 2$ ), 4.25,4.47 (2m, 2H, H-52,H-522), 5.11 (brs, $1 \mathrm{H}, \mathrm{NH}), 5.17(\mathrm{~m}, 1 \mathrm{H}, \mathrm{H}-42), 5.21(\mathrm{~m}, 1 \mathrm{H}, \mathrm{H}-32)$, $5.26(\mathrm{~m}, 1 \mathrm{H}, \mathrm{H}-22), 5.32(\mathrm{dd}, 1 \mathrm{H}, \mathrm{J}=3.2,6.2 \mathrm{~Hz}, \mathrm{H}-$ 12), $5.90(\mathrm{~d}, 1 \mathrm{H}, \mathrm{J}=8.8 \mathrm{~Hz}$, oxadiazoline $\mathrm{H}-6), 7.20$ $7.560(\mathrm{~m}, 4 \mathrm{H}, \mathrm{Ar}-\mathrm{H})$.

General procedures for the reaction of (4) with aromatic aldehydes to afford Schiff2s bases 8(a-d).

A solution of $4(0.01 \mathrm{~mol})$, an aromatic aldehyde $(0.01 \mathrm{~mol})$ in abs. ethanol $(30 \mathrm{ml})$ and glacial acetic acid (1 ml) was refluxed for 6-8 $\mathrm{h}$ (TLC). The solvent was evaporated under reduced pressure and the residue was filtered off and recrystallized from ethanol to afford 8(a-d) in 88-94 $\%$ yields. 
2-((1H-benzo[d]imidazol-2-yl)thio)-N'benzylideneacetohydrazide ( $8 \mathrm{a})$

Yellow gum (88\%), $\mathrm{R}_{\mathrm{f}}=0.72(5 \% \mathrm{MeOH}$ in $\left.\mathrm{CH}_{2} \mathrm{Cl}_{2}\right) .{ }^{1} \mathrm{H}$ NMR (DMSO-d $\left.\mathrm{d}_{6}\right): \delta=4.18\left(\mathrm{~s}, 2 \mathrm{H}, \mathrm{CH}_{2}\right)$, 5.55 (brs, $1 \mathrm{H}, \mathrm{NH}$ ), 7.23-7.85 (m, 9H, Ar-H),8.13 (s, $1 \mathrm{H}, \mathrm{CH}), 8.45$ (brs, $1 \mathrm{H}, \mathrm{NH})$.

2-((1H-benzo[d]imidazol-2-yl)thio)-N'-(2hydroxybenzylidene)acetohydrazide ( $8 \mathrm{~b}$ ).

Yellow gum (90\%), $R_{f}=0.72(5 \% \mathrm{MeOH}$ in $\mathrm{CH}_{2} \mathrm{Cl}_{2}$ ). ${ }^{1} \mathrm{H}$ NMR (DMSO- $\left.\mathrm{d}_{6}\right): \delta=4.15$ (s, 2H, CH2), 5.10 (brs, $1 \mathrm{H}, \mathrm{NH}), 5.34(\mathrm{~s}, 1 \mathrm{H}, \mathrm{OH}), 7.05-7.65(\mathrm{~m}$, $8 \mathrm{H}, \mathrm{Ar}-\mathrm{H}), 8.00$ (brs, $1 \mathrm{H}, \mathrm{NH}), 8.95(\mathrm{~s}, 1 \mathrm{H}, \mathrm{CH})$.

2-((1H-benzo[d]imidazol-2-yl)thio)-N'-(furan-2ylmethylene)acetohydrazide ( $8 \mathrm{c}$ ).

White crystals (92\%), m.p. $160-164{ }^{\circ} \mathrm{C} . \mathrm{R}_{\mathrm{f}}$ $=0.72\left(5 \% \mathrm{MeOH}\right.$ in $\left.\mathrm{CH}_{2} \mathrm{Cl}_{2}\right) .{ }^{1} \mathrm{H}$ NMR $\left(\mathrm{DMSO}-\mathrm{d}_{6}\right): \delta$ $=4.20\left(\mathrm{~s}, 2 \mathrm{H}, \mathrm{CH}_{2}\right), 5.15(\mathrm{brs}, 1 \mathrm{H}, \mathrm{NH}), 6.50-7.80$ $(\mathrm{m}, 7 \mathrm{H}, \mathrm{Ar}-\mathrm{H}), 7.95$ (brs, 1H, NH), $8.42(\mathrm{~s}, 1 \mathrm{H}, \mathrm{CH})$

2-((1H-benzo[d]imidazol-2-yl)thio)-N'-(4(dimethylamino)benzylidene)acetohydrazide (8d).

White crystals (94\%), m.p. $158-160 \stackrel{\circ}{\circ}$. Rf $=0.72(5 \% \mathrm{MeOH}$ in $\mathrm{CH} 2 \mathrm{Cl} 2)$. $1 \mathrm{H}$ NMR (DMSOd6): $\delta=3.10\left(\mathrm{~s}, 6 \mathrm{H}, 2 \mathrm{CH}_{3}\right), 4.18(\mathrm{~s}, 2 \mathrm{H}, \mathrm{CH} 2), 5.11$ (brs, 1H, NH), 6.58-7.64 (m, 9H, Ar-H), 8.05 (brs, $1 \mathrm{H}, \mathrm{NH}), 8.37(\mathrm{~s}, 1 \mathrm{H}, \mathrm{CH})$.
General procedure for preparation of oxadiazoline derivatives 9 (a- d).

A mixture of the shiff bases $8(a-d)(10$ $\mathrm{mmol})$, were dissolved in acetic anhydride $(10 \mathrm{ml})$ and the reaction mixture was refluxed at $100{ }^{\circ} \mathrm{C} 5$ $7 \mathrm{~h}$ (TLC). The mixture was onto crushed-ice and the product that separated out was filtered off, washed with water, and then dried. The products were recrystallized from methanol to give $9(a-d)$ in 80-88 yields.

1-(5-(((1H-benzo[d]imidazol-2-yl)thio)methyl)-2phenyl-1,3,4-oxadiazol-3(2H)-yl)ethanone (9a). Yellow powder $(80 \%)$ m.p. $152-154{ }^{\circ} \mathrm{C} . \mathrm{R}_{\mathrm{f}}$ $=0.72\left(5 \% \mathrm{MeOH}\right.$ in $\left.\mathrm{CH}_{2} \mathrm{Cl}_{2}\right) \cdot{ }^{1} \mathrm{H}$ NMR $\left(\mathrm{DMSO}-\mathrm{d}_{6}\right): \delta$ $=2.10\left(\mathrm{~s}, 3 \mathrm{H}, \mathrm{COCH}_{3}\right), 4.50(\mathrm{~s}, 2 \mathrm{H}, \mathrm{CH} 2), 5.13(\mathrm{brs}$, $1 \mathrm{H}, \mathrm{NH}), 6.55(\mathrm{~s}, 1 \mathrm{H}, \mathrm{CH}) 7.24-7.57(\mathrm{~m}, 9 \mathrm{H}, \mathrm{Ar}-\mathrm{H})$.

1-(5-(((1H-benzo[d]imidazol-2-yl)thio)methyl)-2(2-hydroxyphenyl)-1,3,4-oxadiazol-3(2H)yl)ethanone (9b).

Yellow powder (84\%) m.p. $136-138{ }^{\circ} \mathrm{C} . \mathrm{R}_{\mathrm{t}}$ $=0.72\left(5 \% \mathrm{MeOH}\right.$ in $\left.\mathrm{CH}_{2} \mathrm{Cl}_{2}\right) \cdot{ }^{1} \mathrm{H}$ NMR (DMSO- $\left.\mathrm{d}_{6}\right)$ : $\delta=2.12\left(\mathrm{~s}, 3 \mathrm{H}, \mathrm{COCH}_{3}\right), 4.35$ (s, 2H, CH2), 5.11 (brs, $1 \mathrm{H}, \mathrm{NH}), 5.37(\mathrm{~s}, 1 \mathrm{H}, \mathrm{OH}), 6.95(\mathrm{~s}, 1 \mathrm{H}, \mathrm{CH}) 6.85-$ $7.56(\mathrm{~m}, 8 \mathrm{H}, \mathrm{Ar}-\mathrm{H})$.

Table : 1 Antibacterial activity of different synthesized compounds.

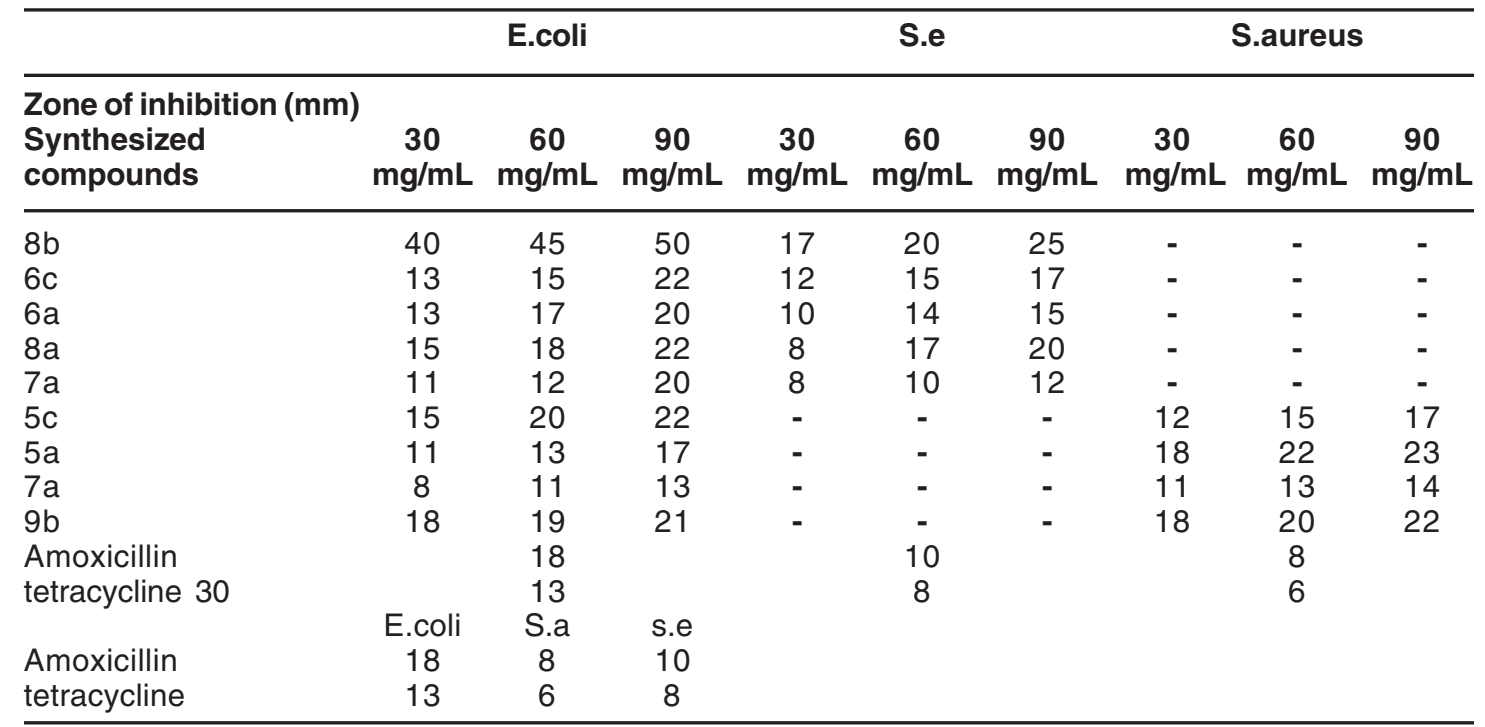


1-(5-(((1H-benzo[d]imidazol-2-yl)thio)methyl)2-(furan-2-yl)-1, 3, 4-oxadiazol-3(2H)yl)ethanone (9c).

Yellow powder (85\%) m.p. $142-144{ }^{\circ} \mathrm{C}$. Rf $=0.72(5 \% \mathrm{MeOH}$ in $\mathrm{CH} 2 \mathrm{Cl} 2)$. $1 \mathrm{H}$ NMR (DMSOd6): $\delta=2.09$ (s, 3H, $\mathrm{COCH} 3), 4.40$ (s, 2H, CH2), 5.14(brs, $1 \mathrm{H}, \mathrm{NH}), 6.87(\mathrm{~s}, 1 \mathrm{H}, \mathrm{CH}), 6.35-7.69(\mathrm{~m}$, $7 \mathrm{H}, \mathrm{Ar}-\mathrm{H})$.

1-(5-(((1H-benzo[d]imidazol-2-yl)thio)methyl)-2(4-(dimethylamino)phenyl)-1,3,4-oxadiazol-3(2H)yl)ethanone (9d).

White crystals (88\%), m.p. $170-172{ }^{\circ} \mathrm{C}$. Rf $=0.72(5 \% \mathrm{MeOH}$ in $\mathrm{CH} 2 \mathrm{Cl} 2)$. $1 \mathrm{H}$ NMR (DMSOd6): $\delta=3.12\left(\mathrm{~s}, 6 \mathrm{H}, 2 \mathrm{CH}_{3}\right), 2.10$ (s, 3h, $\left.\mathrm{COCH}_{3}\right), 4.50$ (s, 2H, CH2), 5.14 (brs, $1 \mathrm{H}, \mathrm{NH}), 6.65(\mathrm{~s}, 1 \mathrm{H}, \mathrm{CH})$ 6.27-7.66 (m, 8H, Ar-H).

\section{MATERIALS AND METHODS}

\section{Antibacterial sensitivity test:}

Microbiological investigation hole well method the investigated isolates of bacteria were seeded in tubes with nutrient broth (NB) two different bacteria species were used Escherichia coli (E.coli), Staphylococcus epidermidis (S. epidermidis) and Staphylococcus aureus (S.aureus). antimicrobial activity of chemical compounds was evaluated by Hole well method, Briefly ,inoculum containing approx. cell density $(1.5 * 108 \mathrm{CFU} / \mathrm{ml})$ was spread on Mueller-Hinton agar (MHA) plates the holes diameter $(0.5 \mathrm{~cm})$ were done in the cool medium after that $50 \mu$ from different concentration of compounds 10,20 and 30 was applied using a micropipette. After incubation for $24 \mathrm{~h}$ in an incubator at $37^{\circ} \mathrm{C}$ for bacteria the inhibition zone diameters were measured and expressed in $\mathrm{cm}$. The previous procedure was repeated with $30 \mu \mathrm{g} / \mathrm{disc}$ of tetracycline and with ampicillin at $10 \mu \mathrm{g} / \mathrm{disc}^{24}$.

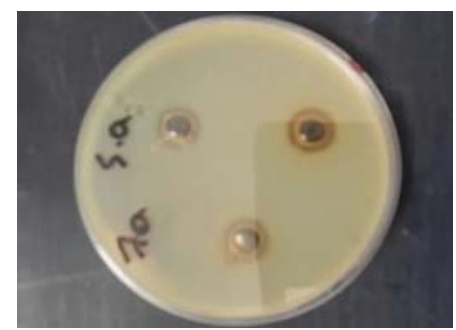

Fig. 1. influence of the synthesized compound 7a on Staphylococcus aureus.
Microbiological investigation of compounds.

Antibacterial of compounds are carried out against the (Gram -ve) as E.coli and (Gram +ve) as S.aureus and (S. epidermidis) , the antimicrobial activity was estimated based on size of inhibition zone around dishes against Gram+v the 7a fig. 1 $5 a, 5 c, 9 a$ fig. 2, 3, 4 and $9 b$ are varied degree of inhibitory effect against S.aureus and $8 a, b c$ and $8 a$ varied degree of inhibitory effect against S.epidermidis ,also in Gram-v the 8b, 6c, 6a, 8a, 7a fig. $5,5 \mathrm{c}$ and $9 \mathrm{~b}$ are varied degree of inhibitory effect against E.coli. The antibacterial activities of compounds against E. coli ,S. aureus and S.epidermids tested and compared to known antibiotics Tables. The results showed that increasing the zone of inhibition in compared with known antibiotic Amoxicillin and tetracycline. the data showed in Table1.

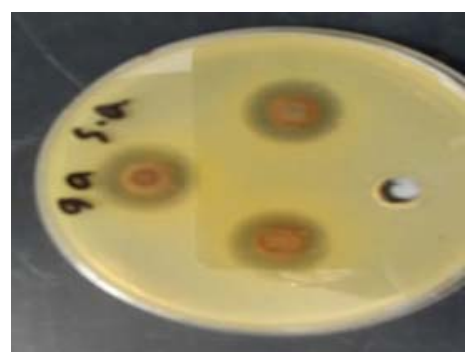

Fig.2. influence of the synthesized compound 9a on Staphylococcus aureus.

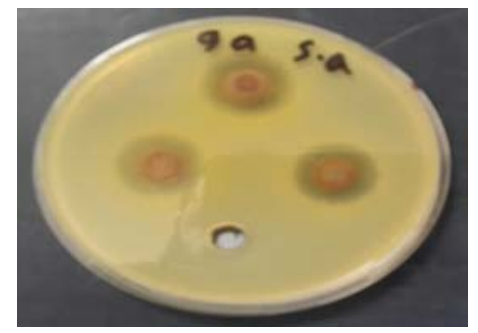

Fig. 3. influence of the synthesized compound 9a on Staphylococcus aureus.

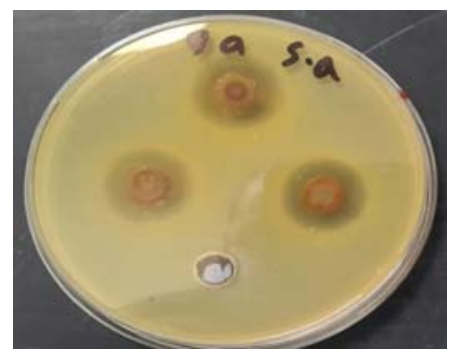

Fig. 4. influence of the synthesized compound 9a on Staphylococcus aureus. 


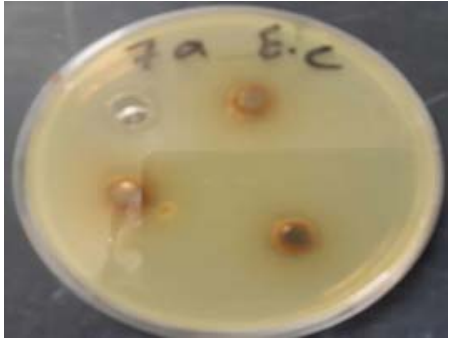

Fig. 5. influence of the synthesized compound $7 \mathrm{a}$ on E.coli.

\section{CONCLUSION}

In this research the 2-mercaptobenzimidazole derivatives were synthized and then were coupled with different sugars ( arabinose, glucose, mannose and arabinose ) and aromatic aldehydes to give the corresponding sugar hydrazones and arylidene derivatives respectively. The synthized compounds were showed high to moderate activity against the (Gram ve) as E.coli and ( Gram +ve) as S.aureus and (S. epidermidis).

\section{ACKNOWLEDGMENTS}

The authors are pleased to acknowledge Taif University for providing the facilities for the research.

\section{REFERENCES}

1. Vikash, K. C.; Devender, P.; Pushpendra, K.; Vijay, Y. Der Pharmacia Lettre. 2016, 8(3), 127-134.

2. Najim, A. M.; Nadhir, N. A.; Layla, J. A.; Sadiq, J. B.; Christophe, P. Z. Naturforsch. 2011, 66, 953-960

3. Chavan, B. B.; Chitte, P. D.; Choudhary, N. P.; Albhar, K. G.; Hukkeri, V. I. International Journal of Scientific Research and Reviews. 2012, 1(3), 22-30.

4. Kiran, M. K.; Sagar, A. J.; Pramod, B. P.; Vikas, R. D.; Shitalkumar, S. P. Der Pharma Chemica. 2016, 8(4), 1-5.

5. Hamiduzzaman, M. d.; Mannan, S. J.; Dey, A.; Abdur Rahman, S. M. Der Pharmacia Lettre. 2014, 6(1), 47-53.

6. Saumya, G.; Surya, P. G.; Neeraj, U.; Gopal, G. Journal of Drug Design and Medicinal Chemistry. 2015, 1(2), 12-16.

7. Anandarajagopal, K.; Ravi, N. T.; Venkateshan, N.; Pooshan, G. V.; Promwichit, P. J. Chem. Pharm. Res. 2010, 2(3), 230-236

8. Kiran, M. K, Sagar, A. J.; Pramod, B. P.; Vikas R. D.; Shitalkumar, S. P. Der Pharma Chemica. 2016, 8(4), 1-5.

9. Saumya, G.; Surya, P. G.; Neeraj, U.; Gopal, G. Journal of Drug Design and Medicinal Chemistry. 2015, 1(2), 12-16.

10. Kumar, D. M.; Dubey, P. K. Indian journal of chemistry. 2012, 51 , 1619-1622.
11. Heralagi, R. V.; Jayaveera, K. N.; Shivkumar, B. Research Journal of Pharmaceutical, Biological and Chemical Sciences. 2012. 3(2), 407-414.

12. Neha, P. V.; Suganthi, V.; Gowri, S. Der Pharma Chemica. 2013, 5(2),139-143

13. Gigani, Y.; Jadhav, S. Int J Pharm Bio Sci. 2010, 1(4), 281-286.

14. Kaur, P.; Wakode, S. R. International Journal of Science and Research. 2016, 5(3), 762772.

15. Zygmunt, K.; Jacqueline, A. U.; Peter, U.; Agata, G.; Bohdan, S.; Agnieszka, L. Acta Biochemica Polonica. 2002, 49(1), 185-195

16. Gangula, M. R.; Yellu, N. R.; Baru, V. K. International journal of applied biology and pharmaceutical technology. 2013, 4(1), 3846 .

17. Sahu, S. K.; Banerjee, M.; Samantray, A.; Behera, C.; azam, M. A. Tropical Journal of Pharmaceutical Research. 2008, 7(2), 961968.

18. Ramanpreet, W.; edaitullah, M. d.; Syeda, F. N.; Khalid, I.; Lamba, H. S. International Journal of Research in Pharmacy and Chemistry. 2011, 1(3), 565-574.

19. Singh, G.; Kaur, M.; Chander, M.; Rayat, B. International Research Journal of Pharmacy. 2013, 4(1), 82-87.

20. Suraj, B. A.; Deshp, M. N.; Kolhatkar, D. G. Int 
J Pharm Bio Sci. 2012, 3(2), 6-11.

21. Reddy, C. M.; Jayakar, B.; Srinivasan, R. Int J Pharm Bio Sci. 2010, 1(4), 81-86.

22. Ansari, K. F, Lal, C. European Journal of Medicinal Chemistry. 2009, 44(10), 40284033.

23. Kus, C.; Ayhan-Kýlcýgil, G.; zbey, S. O.; Kaynak, F.; Kaya, M.; Can-Eke, B. Bioorganic
\& Medicinal Chemistry. 2008, 16(8), 42944303.

24. Majali, I. S.; Oran, S. A.; Khleifat, K. M.; Qaralleh, H.; Rayyans, W. A.; Althunibat, O. Y. African Journal of Microbiology Research. 2015, 9(51), 2410-2414. 